\title{
Welcome to In vitro models
}

\author{
J. Miguel Oliveira ${ }^{1,2} \cdot$ Ketul C. Popat $^{3}$
}

Received: 5 July 2021 / Revised: 8 August 2021 / Accepted: 23 August 2021 / Published online: 28 October 2021

(c) The Author(s), under exclusive licence to Springer Nature Switzerland AG 2021

\section{Welcome, mission and vision}

The cross-disciplinary field of in vitro models has been attracting a great deal of attention in the last decade due to the need to address the paradigm-shifts in research related to:

- An increasing trend for "saying goodbye to flat biology", i.e. introduce 3D in research methods in order to develop tools that can better emulate human tissues/organs and diseases;

- The need to reduce/eliminate extensive animal experimentation.

In vitro models is a hybrid journal published by Springer (part of Springer Nature), and it is the first of the kind focusing on research that deals with the upcoming and impactful field of in vitro models. With Springer Nature, a global publisher dedicated to providing the best possible service to the whole research community, In vitro models will be able to reach a vast audience and target a multidisciplinary research community.

The In vitro models mission is to contribute to the fast peer-review and cross-disciplinary dissemination and foster the application of research and technology developments in the field of in vitro models. Our vision is to establish and

J. Miguel Oliveira

miguel.oliveira@i3bs.uminho.pt

1 3B's Research Group, I3Bs - Research Institute on Biomaterials, Biodegradables and Biomimetics, University of Minho, Headquarters of the European Institute of Excellence on Tissue Engineering and Regenerative Medicine, AvePark, Zona Industrial da Gandra, 4805-017 Barco, GMR, Portugal

2 ICVS/3B's - PT Government Associate Laboratory, Braga/Guimarães, Portugal

3 Department of Mechanical Engineering/School of Biomedical Engineering/School of Advanced Materials Discovery, Colorado State University, Fort Collins, CO 80523, USA become a leading scientific journal with worldwide coverage for the successful interdisciplinary integration of research knowledge and diffusion of significant findings related to the in vitro models research. The science related to in vitro models is currently receiving enormous interest from different disciplines, worldwide, from drug development and delivery, biofabrication, materials science, nanomedicine, microfluidics, personalized medicine, biology, cancer research, and tissue engineering. Therefore, we also aim to foster the growth of the scientific community in this field, in the coming years.

The Editors-in-chief of this new journal are Prof. J. Miguel Oliveira and Prof. Ketul Popat.

Prof. J. Miguel Oliveira is a biochemist, and received his Ph.D. in Materials Science and Technology, Knowledge Area of Tissue Engineering-Hybrid Materials from University of Minho, Braga-Portugal, in 2009. He is a Principal Investigator with Habilitation and Professor in Doctoral Program in Tissue Engineering, Regenerative Medicine and Stem Cells (TERM\&SC) at University of Minho, Guimarães-Portugal. He is the Vice-President of I3Bs-Institute for Biomaterials, Biodegradables and Biomimetics (Univ. Minho) and Director of Pre-Clinical Research at the FIFA Medical Center, Estádio do Dragão, Porto, PT, since Feb. 2013. Over the years, he has focused his work on the field of biomaterials for tissue engineering, nanomedicine, stem cells, and cell/drug delivery applications and established a research line within the I3Bs on 3D in vitro models. As a result of his proficiency, he has published so far more than 400 scientific contributions in scientific journals with the referee. He has approved more than 20 patents, published 6 books, 5 special issues in scientific journals, and more than 100 book chapters in books with international circulation. $\mathrm{He}$ has been awarded several prizes including the prestigious "Investigador FCT 2012 and Investigador FCT 2015" attributed by FCT (Portugal), and The Jean Leray Award 2015 from European Society for Biomaterials for Young Scientists for Outstanding Contributions within the field of Biomaterials. He is also a member of the National Ethics Committee for Clinical Trials from SNS-Serviço Nacional de Saúde (Portugal). 
Prof. Ketul C. Popat a Professor in the Department of Mechanical Engineering/School of Biomedical Engineering/ School of Advanced Materials Discovery at Colorado State University. He is also Director of Undergraduate Programs in Biomedical Engineering. Prior to that, he was working as a Research Specialist in the Department of Physiology at University of California, San Francisco. His research expertise is in the areas of biomaterials, tissue engineering, and nanostructured surfaces for medical devices. He has authored over 140 peer-reviewed publications in highimpact journals and presented his work at numerous national and international level conferences. He received his Ph.D. in Bioengineering from University of Illinois at Chicago in 2003, M.S. in Chemical Engineering from Illinois Institute of Technology, Chicago, in 2000, and B.E. in Chemical Engineering from M. S. University in India in 1998.

\section{The senior honorary advisors, editorial board, and young editorial board}

The In vitro models gathers an outstanding Editorial Board composed of two Senior Honorary Advisors, Prof. Rui L. Reis and Prof. David Kaplan.

Professor Rui L. Reis, PhD, DSc, Hon. Causa MD, Hon Causa PhD, FBSE, FTERM, member of NAE, FAIMBE, $F E A M B E S$, was born in 1967 in Porto, Portugal. He is a Full Professor of Tissue Engineering, Regenerative Medicine, Biomaterials and Stem Cells at University of Minho (UMinho), Director of the 3B's Research Group-member of the I3Bs - Institute for Biomaterials, Biodegradables and Biomimetics, and Director of the ICVS/3B's Associate Laboratory, both of UMinho. He is also the CEO of the European Institute of Excellence on Tissue Engineering and Regenerative Medicine, the Global Past-President of the Tissue Engineering and Regenerative Medicine International Society (TERMIS) and the Editor-in-chief of the Journal of Tissue Engineering and Regenerative Medicine. He is in the Editorial Board of several other relevant journals. He is a recognized World Expert in the TERM and biomaterials fields that has edited several books and has more 1460 published works listed on ISI Web of Knowledge with an h index of 98 (1360 works and $\mathrm{h}=102$ in Scopus and 2210 and $h=124$ in Google Scholar), being also an inventor of around 115 patents. Based on those, he co-funded several companies that raised important private investments. According to Google Scholar, his work has been cited more than 68,500 times. He has been awarded many important international prizes, including among several others different innovation awards, the Jean Leray and George Winter Awards (ESB), the Clemson Award (SFB), the TERMIS-EU contributions to the literature Award and the TERMIS-EU
Career Achievement Award, and in 2018 the UNESCOInternational Life Sciences Award and the IET A. F. Harvey Engineering Research Prize. He is a member of the National Academy of Engineering (USA), FBSE, FTERM, and was awarded 2 honouris causa degrees. He is the PI of projects with a budget totalizing more than 100 MEuros of which around 50 MEuros are U. Minho funding.

Prof. David Kaplan holds an Endowed Chair, the Stern Family Professor of Engineering, at Tufts University (USA). $\mathrm{He}$ is Professor \& Chair of the Department of Biomedical Engineering and also holds faculty appointments in the School of Medicine, the School of Dental Medicine, Department of Chemistry, and the Department of Chemical and Biological Engineering. His research focus is on biopolymer engineering to understand structure-function relationships, with emphasis on studies related to self-assembly, biomaterials engineering, and functional tissue engineering/regenerative medicine. He has published over 900 peer-reviewed papers, including on a variety of structural proteins. He directed the NIH P41 Tissue Engineering Resource Center (TERC) that involved Tufts University and Columbia University from 2004 to 2019 and continues to lead the biomaterials core (2020-present). He serves on the editorial boards of numerous journals and is Editor-in-chief for ACS Biomaterials Science and Engineering, after serving as an associate editor for Biomacromolecules for 15 years. He has received a number of awards for teaching, was elected Fellow American Institute of Medical and Biological Engineering, elected to the International Academy of Medical and Biological Engineering, and received the Columbus Discovery Medal and the Society for Biomaterials Clemson Award for contributions to the literature.

The Editorial Board (EB) has been set up and already gathers over 40 top researchers from all over the world, and it is expanding. The role of members of the EB involves:

- Helping the journal to attract high-quality manuscripts, for example by mentioning the journal to peers and at conferences;

- Submitting own research and/or writing occasional reviews for the journal;

- Suggesting topics and authors for commissioned reviews and commentaries;

- Providing feedback and suggesting improvements for the journal;

- Reviewing manuscripts for the journal (no more than 3 a year).

In addition, a panel of Early Career Board Members is currently being established. The In vitro models will open a call for other members to join the early career panel, targeting researchers at the early stage in their careers (less than 10 years after completion of their $\mathrm{PhD}$ ). 


\section{Aims, scope, and topics}

The In vitro models aims to become a leading journal among the top multidisciplinary journals covering all aspects of in vitro models' research, which also includes a broad spectrum of topics related to the interface of biomaterials science, chemistry, physics, engineering, informatics, nanotechnology, pharma, medicine, and biology.

The scope of the In vitro models is related to expanding the field of in vitro cell/tissue/organs culture models. A search in the PUBMED database (July 1, 2021) using the keyword "In vitro models" shows that this field has significantly grown as reflected by the number yearly published works; i.e., since the year 2000 until 2020, the number of publications increased from 6599/year to 29,411/year, respectively.

This new journal will provide a specialized forum for the publication of ideas/concepts, and significant and original work related to experimental and theoretical studies on fundamental and applied inter- and multi-disciplinary research dealing with in vitro models, with a particular focus on the following topics:

- In vitro models;

- New perspectives in in vitro tissue models research;

- Complementary and alternative models to animal experimentation;

- Cell culturing methods (e.g., 2D, 3D, and 4D cell culture);

- Ex vivo models;

- Biomaterials as extracellular matrix;

- Drug discovery and development;

- Bioprinting;

- Stem cells and cell engineering;

- Dynamic culturing;

- Biofluid mechanics;

- Microfluidics;

- Organ-on-a-chip;

- Bioreactors;

- In silico models;

- Modelling and simulation;

- Nanomedicine;

- Tissue engineering;

- Precision medicine;

- Cancer research;

- Regenerative medicine;

- Extracellular vesicles development and characterization;

- New in vitro assays development for regulatory approvals;

- Translational research.
The main list of keywords includes the following: 2D, 3D and 4D models; 3D cell culture; Dynamic culturing; Extracellular matrix; In vitro models; In silico models; Biomaterials; Biofluids; Biology; Biological compounds; Bioprinting; Bioreactors, Biomedical science; Cell engineering; Drug development; Ex vivo models; Extracellular vesicles development and characterization; Methods; Modelling; Microfluidics; Pharma; Organoids, Organ-ona-chip; Drug testing; Nanomedicine; New in vitro assays development for regulatory approvals; Tissue engineering; Theoretical studies; Diseases; Tissue models; Tumor models; Stem cells; Scaffolds; Spheroids, Simulation; Standardization.

\section{Types of articles}

The In vitro models journal will peer-review and publish different types of articles, including (i) Original Articles consisting of full-length accounts of original research that provide a comprehensive report of original research presenting a significant advance of lasting impact, related to the topics covered by the journal; (ii) Rapid Communications, where authors must provide short articles reporting outstanding, new, and timely developments/contributions within the scope of the Journal. Length should be no longer than 5 journal pages or 4500 words, including figures; (iii) Reviews that critically evaluate an existing work of multiple groups in a field or topic related to the scope of the journal. It should reflect the current state-ofthe-art and be of general interest to the readership. The structure and length should follow the standard Springer instructions for authors. Length is flexible (6-20 or more pages) and a maximum of 400 references; (iv) Concepts where authors should provide a brief paper with key details about new ideas, research concepts, or findings, related to in vitro models, that are of particular interest to a vast audience, and that merit further exploration. Length should be 3-5 pages; (v) Methods focusing on rapidly developing laboratory techniques and disruptive methods/modelling of significance in the in vitro models' field. Length should be no longer than 5 journal pages or 4500 words, including figures; (vi) Opinion Papers by invitation from the Editor(s)-in-Chief. Authors should report original and personal views on a given subject. It should be based on published data, should not expand on opinions by others, and should be written in a logical, professionally sound, and convincing way. Thus, authors should outline, and craft selected arguments by bringing original and groundbreaking ideas and imaginative research solutions. Length should be no longer than 5 pages; (vii) Highlights by invitation from the Editor(s)-in-Chief. Authors should 
report/discuss a particular and impactful research finding. It must contain a brief abstract of $\sim 150$ words and max of up to 10 references. Highlights also include a graphical image. Length should be 2-3 pages; and (viii) Editorials to be submitted by the Editor(s)-in-Chief or other members of the Editorial board. Typical length should be 1-2 pages.

\section{Relevance and potential impact}

The In vitro models aims to represent and publish research to target a multidisciplinary community. The audience comprises the following: biologists, biomedical engineers, chemists and biochemists, physicists, information technologists, materials and nanotechnology scientists, pharma researchers, regulators, and medical doctors. As aforementioned, the new journal covers an expanding and impactful area with increasing funding. The essence of the journal is to publish high-quality papers and disseminate findings and new standardized methods, but also to be a platform for gathering different experts and foster brainstorming across disciplinary bounds to generate new ideas and collaborations, and ultimately have a broad and long-lasting impact.

Data availability Not applicable.

\section{Declarations}

Conflict of interest The authors declare no competing interests. 